\title{
EXPERIMENTAL STUDY OF LAMINAR SEPARATION PHENOMENON COMBINING WITH NUMERICAL CALCULATIONS
}

\author{
Nguyen Manh Hung, Hoang Thi Bich Ngoc \\ Hanoi University of Technology
}

\begin{abstract}
The separation is much more sensitive for laminar flow than for turbulent flow. These remarks have been attested for both subsonic and supersonic flows. However, they are not applicable to transonic flows when there are interactions between boundary layer and shock wave. Along with the Reynolds number, the Mach number is a necessary dimensionless parameter for the condition and the mechanism of separations. The report presents one part of studies on laminar separation with Mach number of incompressible flow. The laminar regimes correspond to flows through wind turbine blades. Our experimental work for laminar separation phenomenon was carried out in a subsonic open circuit wind tunnel by taking photographs. The accuracy of experimental results basically depends on the accuracy of wind tunnel and the quality of smoke on density and constitutive materials. Experimental results permit to determine the position of separation and the form of turbulent region followed from the separation point. Numerical studies were simultaneously realized. Based on obtained experimental and numerical results, the report presents also the comparison between the laminar separation and the turbulent separation.
\end{abstract}

Keywords: Laminar separation, Experimental study, Numerical calculation.

\section{INTRODUCTION}

For subsonic flows over a plane plate or a profile, Reynolds number $\mathrm{Re}=10^{5} \mathrm{can}$ be considered as Reynolds number of transition [1]. With $\operatorname{Re}<10^{5}$, flow is laminar. The Reynolds number characterizes the turbulence. The condition of separation still depends on pressure gradient concerning the geometry and the incidence.

In the same conditions of geometry and incidence, the laminar flow has a separation, but the turbulent flow can have not one. The higher the turbulence ratio is (increased with the Reynolds number), the lower the risk of separation is. However, this remark is only verified for subsonic flows in which there is not the transonic effect. For transonic flows having the interaction between boundary layer and shock wave, it is necessary to take into account the Mach number for investigating the boundary layer separation.

Fig. 1 illustrates numerical results (using viscous Fluent) for profile Naca 4412, incidence angle $\alpha=2.5^{\circ}$ and different free Mach numbers. In case of $\mathrm{M} \sim 0\left(\operatorname{Re}=1.3 \times 10^{4}\right)$, flow is laminar, the separation occurs on profile upper, lift coefficient integrated from 
pressure distribution has value $C_{L}=0.48$. For case of $M=0.3\left(R e=6.8 \times 10^{5}\right)$, flow is turbulent and there not any separation, lift coefficient $\mathrm{C}_{\mathrm{L}}=0.72$. With free Mach number $\mathrm{M}=0.85\left(\mathrm{Re}=1.9 \times 10^{7}\right)$, flow is transonic, a separation occurs due to interaction between boundary layer and shock wave, lift coefficient in this case $\mathrm{C}_{\mathrm{L}}=0.49$.

The report presents results of laminar separation phenomenon in subsonic flows, in which Mach number is considered as zero. The mechanism of separation here is influenced by the pressure gradient law concerning the incidence. The separation phenomenon depends on Mach number will be presented in other report.

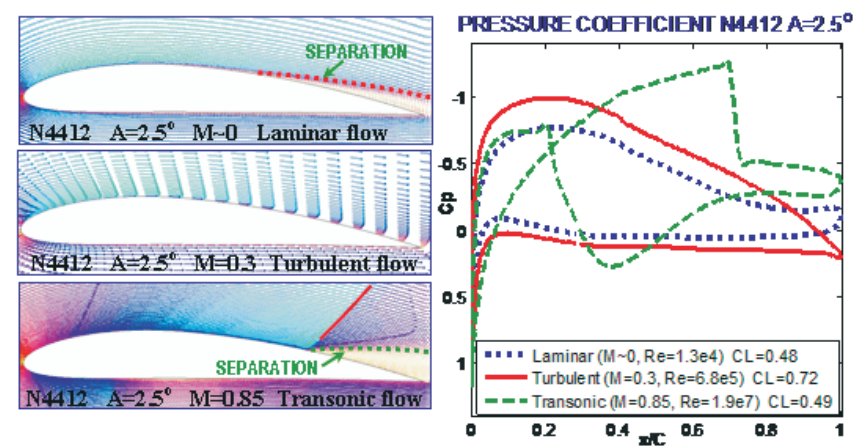

Fig. 1. Separation with different free Mach number

The wind tunnel used for taking experiments is an open circuit wind tunnel (AF6116) having a high accuracy. This wind tunnel was from France and installed at Hanoi University of Technology by production company Dantec Dynamics. The wind tunnel can produce flows with Mach number $\mathrm{M}=0.15$. The test chamber has the cross section $400 \mathrm{~mm} \times 500 \mathrm{~mm}$ and the length of $1000 \mathrm{~mm}$. Experiments were carried out with rectangular wings having spans of $400 \mathrm{~mm}$, profiles Naca 0012 and Naca 4412 located at different angles (corresponding to angles of attack).

Experimental results of laminar flows with separation have been analyzed and compared with numerical results in order to recognize advantages and disadvantages of each method, experimental one or numerical one. Numerical calculations were realized for incompressible turbulent flows to deduce different aspects between the laminar flow separation and the turbulent flow separation.

Our computational programs are for invicid flows which use singularity method and full potential equation method. We also used Fluent software to calculate invicid and viscous cases. Results of invicid flows calculated from Fluent were compared with published results to verify the operation. Results of invicid and viscous Fluent were compared each other.

\section{EXPERIMENTAL RESULTS OF VISUALIZATION}

The rectangular wings of profiles Naca 4412 and Naca 0012 were located at diferrent angles: $0^{\circ}, 2.5^{\circ}, 3.75^{\circ}, 5^{\circ}, 6.25^{\circ}, 7.5^{\circ}, 8.75^{\circ}, 10^{\circ}, 12.5^{\circ}, 15^{\circ}$ with wind speed $\mathrm{V}=2 \mathrm{~m} / \mathrm{s}$. 
Fig. 2 and Fig. 3 show photographs on the interaction between air flow and wings at different angles of attack. A couple of photographs have the same angle, profile Naca 4412 being on the left and profile Naca 0012 being on the right. The separation position is marked by a round point.

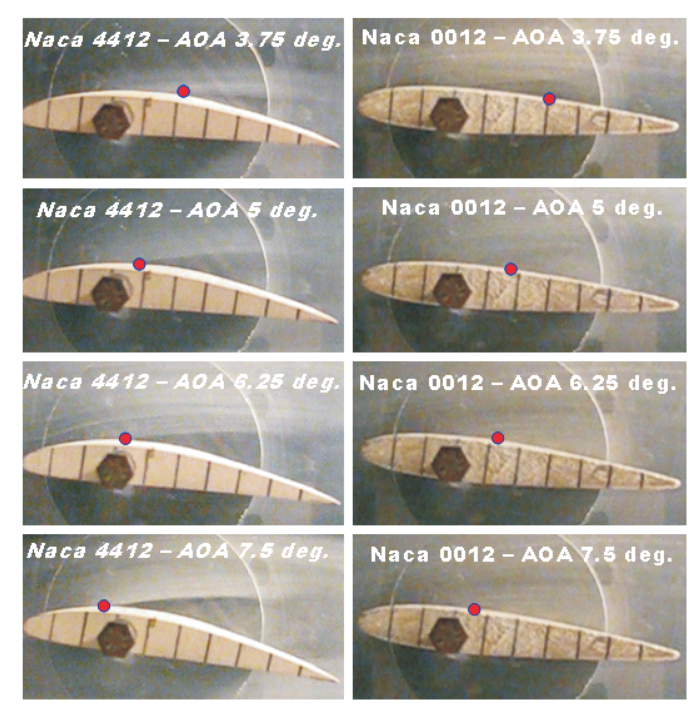

Fig. 2. Photographs at attack angles: $3.5^{\circ}, 5^{\circ}, 6.25^{\circ}, 7.5^{\circ}$
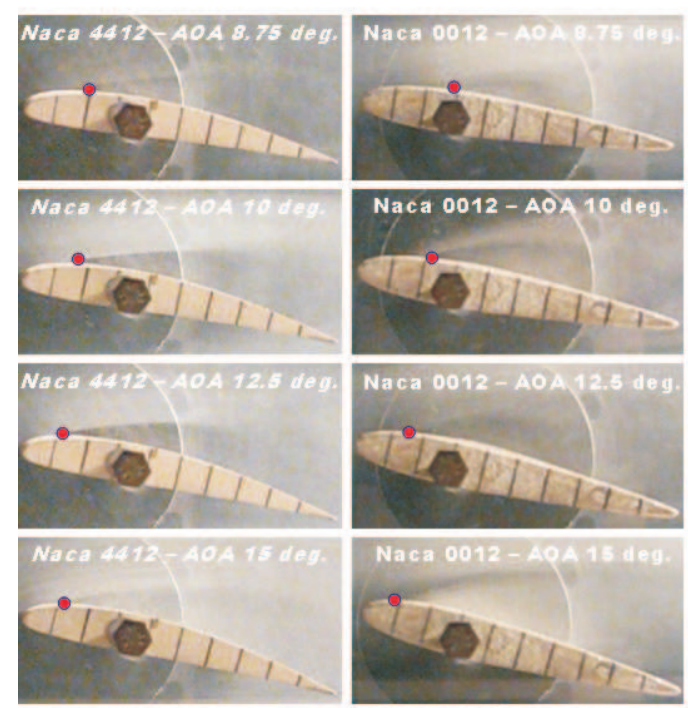

Fig. 3. Photographs at attack angles: $8.75^{\circ}, 10^{\circ}, 12.5^{\circ}, 15^{\circ}$ 


\section{ANALYSIS OF EXPERIMENTAL RESULTS}

\subsection{Observation of laminar flows with separation}

Operating the wind speed $\mathrm{V}=2 \mathrm{~m} / \mathrm{s}$, with the kinematic viscosity of air $\nu=$ $15 \times 10^{-6} \mathrm{~m}^{2} / \mathrm{s}$, the chord length of profile $\mathrm{C}=100 \mathrm{~mm}$, Reynolds number is then: $\mathrm{Re}=$ V.C $/ \nu \approx 13000$. In photos, it is observed that laminar external flow regions are characterized by straight thread layers. From separation point for flow near wall, straight thread layers do not form and they become disordered that take shape a turbulent region. The boundary of the followed turbulent region and the straight thread layers is very clear in photos.

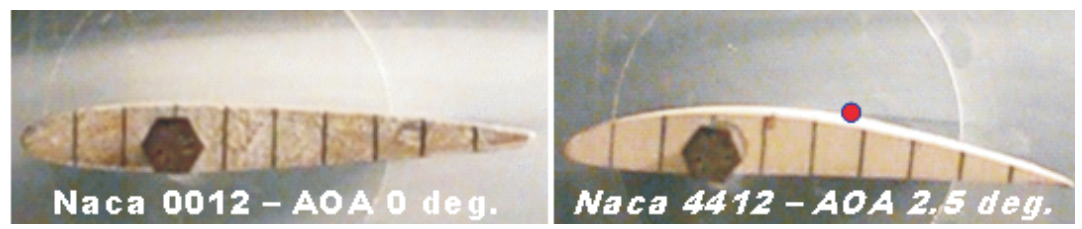

Fig. 4. Flows without separation (Naca 0012, $0^{\circ}$ ) and with separation (Naca 4412, $2.5^{\circ}$ )

In Fig. 4, for profile Naca 0012 with the incidence of $0^{\circ}$, laminar flow is covered all the profile contour with straight thread layers, without boundary layer separation. While for profile Naca 4412 having the incidence of $2.5^{\circ}$, the separation occurs at position $\mathrm{x} / \mathrm{C}$ $=0.57$ of upper contour from the leading edge.

By observing the position of separation in figures, we can note some points:

- For each profile, Naca 4412 or Naca 0012, along with the increase of incidence, the separation position is displaced to the leading edge.

- Separation positions are different for profile Naca 4412 and Naca 0012. However, it is impossible to judge that at the same angle of attack the separation position of profile Naca 4412 is always ahead in comparison with one of profile Naca 0012. This thing depends on the incidence, which will be analyzed in next part.

\subsection{Analysis of phenomena}

As we know, for subsonic aerodynamic profiles, the lift strongly decreases when the boundary layer separation occurs at great angles of incidence (for profile Naca 0012, this angle of incidence is 12 degrees). The rules are true for turbulent flows. For laminar flows, we will analyze experimental results to find different effects for turbulent flow and laminar flow.

Fig. 3 shows the separation at a very small incidence, at $2.5^{\circ}$ for profile Naca 4412 with a turbulent region followed from the separation point that takes $43 \%$ of upper contour. It is observed that for profile Naca 4412 at $5^{\circ}$ (in Fig. 2), the separation is followed by a large turbulent region taken more $60 \%$ of upper contour. From the experimental results, it is possible to notice that the laminar flow is very sensitive to the separation.

It is evident that the separation position and the form of followed turbulent region depend on still the geometry. For angles of attack smaller than $10^{\circ}$, the separation for profile Naca 4412 is stronger than one for profile Naca 0012 (in aspect of followed turbulent 
region form). However, for angles of $10^{\circ}, 12.5^{\circ}, 15^{\circ}$, the thing is inverse. It is due to different curvatures of upper contours of profile Naca 4412 and profile Naca 0012.

Despite efforts on experimental conditions, results were not always expected. Many results were been rejected due to disturbances. In this work, if putting by the wind tunnel accuracy, experimental results still depend on the smoke quality and the camera position that can give bad photos. In next part, we will bring into comparison experimental results and numerical results.

\section{COMPARISON BETWEEN EXPERIMENTAL AND NUMERICAL RESULTS}

The boundary layer separation is a singular phenomenon. For this reason, a numerical method for calculating flows with separation is not always evident. Our codes are based on compressible flow theory but only invicid one, which are unable to calculate flows with separation. Fluent software can be used for these cases. However, exploitations and operations of Fluent to obtain acceptable results are not always clear. For incompressible flows without separation, pressure coefficients are not very different between viscous and invicid flow calculations [4]. Therefore, in order to ensure good exploitations of Fluent, it is necessary to compare results calculated by Fluent and by other methods for incompressible flows.

\subsection{Comparison between results calculated by Fluent and by other methods}

Fluent can be operated for calculating viscous and invicid flows. Before using grids and correctional factors in Fluent for calculations, these ones were attested to some cases. Results of Fluent have been compared with results calculated from our code programmed by full potential equation method. The equation is as follows:

$$
\left[a^{2}-\left(\frac{\partial \Phi}{\partial x}\right)^{2}\right] \frac{\partial^{2} \Phi}{\partial x^{2}}+\left[a^{2}-\left(\frac{\partial \Phi}{\partial y}\right)^{2}\right] \frac{\partial^{2} \Phi}{\partial y^{2}}-2\left(\frac{\partial \Phi}{\partial x}\right)\left(\frac{\partial \Phi}{\partial y}\right) \frac{\partial^{2} \Phi}{\partial x \partial y}=0
$$

where $\Phi$ is full velocity potential, and components of velocity: $u=\frac{\partial \Phi}{\partial x}, v=\frac{\partial \Phi}{\partial y}, a$ is speed of sound.

The algorithm and the resolution of full potential equation (FPE) have been presented in [3]. This program was verified for the calculation of subsonic flows (incompressible, compressible and transonic flows).

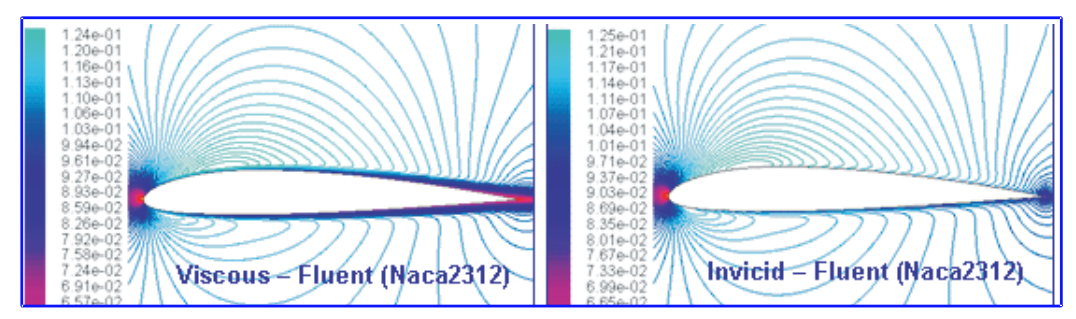

Fig. 5. Iso-Mach lines - Naca 2312, AOA $0^{\circ}, \mathrm{M}=0.1$ 
Fig. 5 shows different results on iso-Mach lines calculated by viscous Fluent and invicid Fluent for flow around profile Naca 2312 with free Mach number $\mathrm{M}=0.1$ and the incidence of $0^{\circ}$. Differences are only in boundary layer, but results are similar in external flow. For the incompressible flow, pressure coefficients are similar for viscous and invicid models of Fluent and that is presented in Fig. 6. Figure 6 also shows pressure coefficient calculated from established program FPE. It is clear that the three results (viscous Fluent, invicid Fluent, invicid FPE) are almost similar, have not considerable differences. Fig. 7 represents the comparison between numerical result of pressure coefficient calculated from present FPE program and experimental result of Riegels [6] (for profile Naca 0012 , incidence of $0^{\circ}$, incompressible flow), and our experimental results [5]. The above comparisons verify application operations of Fluent software for viscous and invicid incompressible flows.

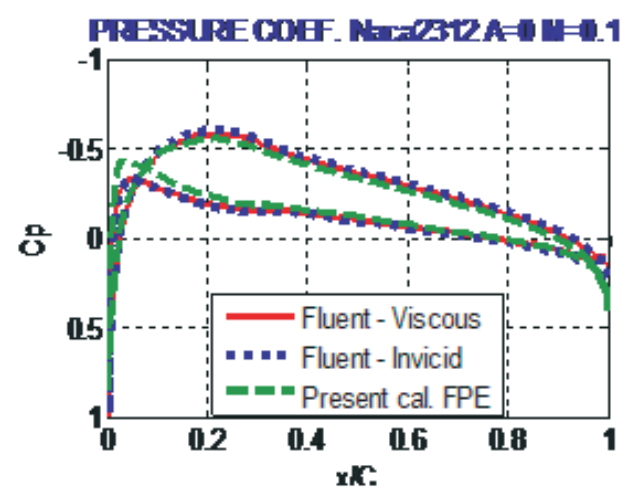

Fig. 6. Cp - Comparison: Viscous, invicid Fluent and invicid FPE

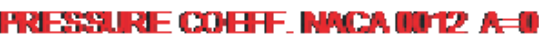

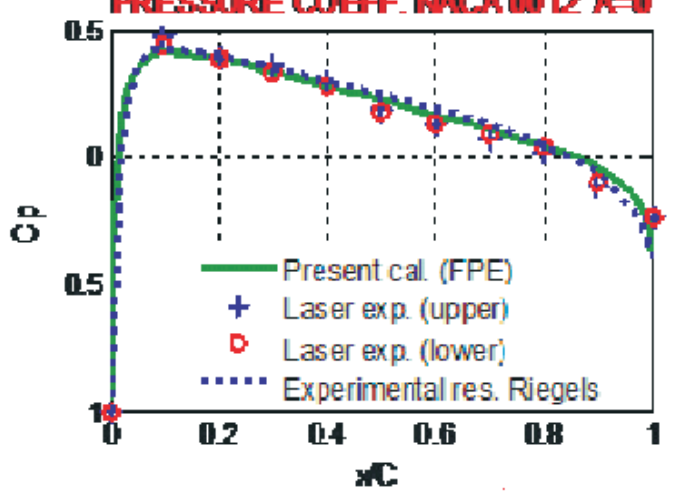

Fig. 7. Cp - Comparison: Experiments [5], [6] and present FPE 


\subsection{Comparison between results of experiment and simulation}

Fig. 8 , with sub-figures on the right, shows results calculated by Fluent on velocity fields for profile Naca 4412 at attack angles of $7.5^{\circ}, 6.25^{\circ}, 3.75^{\circ}, 2.5^{\circ}$, and Reynolds number $\mathrm{Re}=1.3 \times 10^{4}$ (velocity at infinity $\mathrm{V}_{\infty}=2 \mathrm{~ms}^{-1}$. Corresponding sub-figures on the left represent experimental photographs with the position of separation and the boundary line of followed turbulent region. It is firstly observed when comparing experimental results with numerical results that the similarity on points of separation. The boundary between laminar external flow and turbulent region followed from separation point is clear in photographs of experiment (which is outlined by dash line).

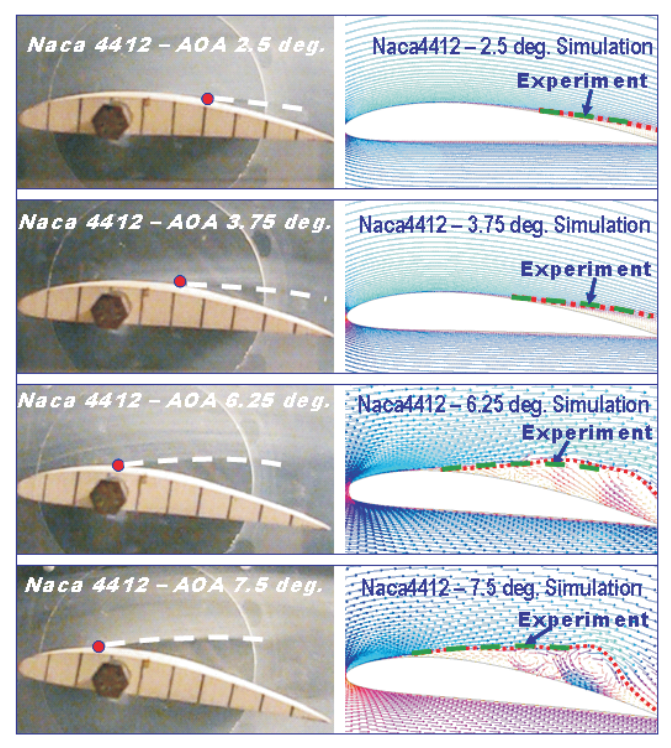

Fig. 8. Results of experiment and simulation (AOA: $\left.2^{\circ}, 3.75^{\circ}, 6.25^{\circ}, 7.5^{\circ}\right)$

In order to compare the laminar separation and the turbulent separation, consider the case of profile Naca 4412 with the incidence of $10^{\circ}$. The turbulent flow was calculated for Mach number $\mathrm{M}=0.3$ that is corresponding to Reynolds number $\mathrm{Re}=7 \times 10^{5}$. In Fig. 9, numerical results show that there is a weak separation near the trailing edge (at $\mathrm{x} / \mathrm{C}=0.95$ ). While, the laminar separation in Fig. 7 (at the incidence of $10^{\circ}$ ) is very strong at the position $\mathrm{x} / \mathrm{C}=0.16$. Fig. 9 shows also the pressure coefficient calculated by four methods: viscous Fluent, invicid Fluent, invicid FPE program, invicid program of singularity method [2]. On lower contour, there was not the separation and thus the three invicid results and the one viscous result are similar. On upper contour, due to the weak separation near the trailing edge, the viscous result is a little different from the invicid results.

For profile Naca 4412, the boundary layer separation of incompressible turbulent flow occurs at the incidence of $10^{\circ}$ and from this angle of incidence the lift coefficient decreases (for angles of incidence being greater than $10^{\circ}$, lift coefficient curve becomes 


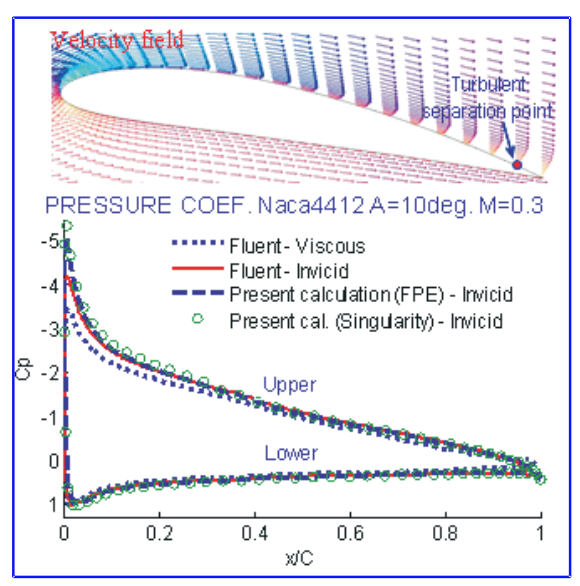

Fig. 9. Pressure coefficient - Comparison: present codes, invicid Fluent, viscous Fluent

non-linear). For profile Naca 0012, the critical attack angle for non-linear zone of lift coefficient curve is $12^{\circ}$ (see Fig. 11).

\section{CONCLUSIVE RESULTS OF EXPERIMENT ON POSTIONS OF LAMINAR SEPARATION}

Fig. 10 shows graphs of experimental results on positions of laminar separation for profile Naca 4412 and profile Naca 0012 depending on the incidence with Reynolds number $\mathrm{Re}=1.3 \times 10^{4}$. Two graphic curves for Naca 4412 and Naca 0012 have different variations. At the incidence of $14^{\circ}$, the two curves intersect. Therefore, for angles of incidence being smaller than $14^{\circ}$, separation point of profile Naca 4412 is ahead of one of profile Naca 0012. And for angles of incidence being greater than $14^{\circ}$, the thing is inverse.

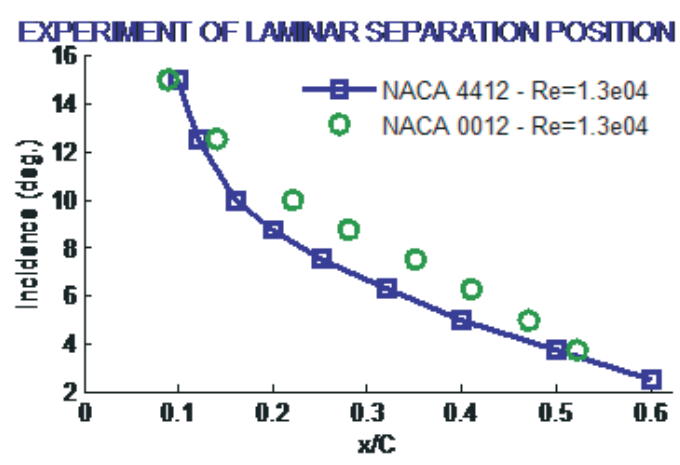

Fig. 10. Positions of laminar separation (upper side)

In order to compare differences on positions of separation for laminar and turbulent flows, numerical calculations were carried out for incompressible flow with free Mach number $\mathrm{M}=0.3$ (i.e. $\operatorname{Re}=7 \times 10^{5}$ ). 


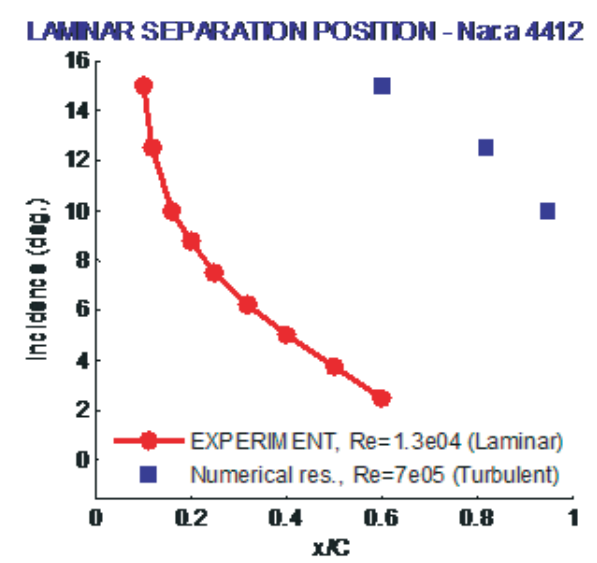

Fig. 11. Laminar and turbulent separation positions - Naca 4412

It is observed that for profile Naca 4412, turbulent separations occur at three angles of incidence $15^{\circ}, 12.5^{\circ}, 10^{\circ}$ with its positions being nearer the trailing edge (see Fig. 11). And for profile Naca 0012, turbulent separations occur at two angles of incidence $15^{\circ}$ and $12.5^{\circ}$. At $15^{\circ}$, turbulent separation is very strong and occupies $80 \%$ of upper contour (see Fig. 12).

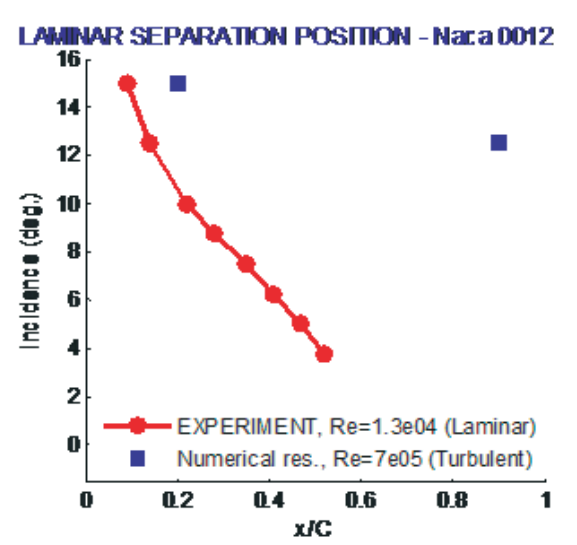

Fig. 12. Laminar and turbulent separation positions - Naca 0012

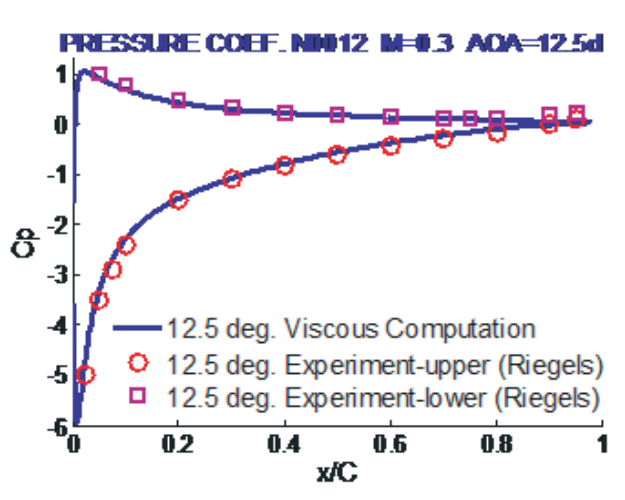

Fig. 13. Comparison between numerical and experimental results of turbulent flow - Naca 0012, $\alpha=12.5^{\circ}$

For profile Naca 4412 with angles of incidence being smaller than $10^{\circ}$, incompressible turbulent flows have not separations. For profile Naca 0012, there are not separations with angles of incidence being smaller than $12^{\circ}$. While computational calculations of laminar lows with boundary layer separation have weak convergence, the convergence is good for turbulent flows with separation. The accuracy of turbulent flow calculations is shown in Fig. 13 by comparison with experimental results F. W. Riegels [6] on pressure coefficient 
of profile Naca 0012 at angle of incidence of $12.5^{\circ}$ (the case has a separation on the upper of profile in Fig. 12). The turbulent property diminishes the risk of separation.

\section{CONCLUSION}

The experimental work combining with numerical calculations permits to deduce some following remarks:

- Separation positions of the considered laminar flows are similar when comparing experimental results and numerical results.

- Laminar flow regime is very sensitive to the boundary layer separation, much more than in comparison with turbulent flow regime.

- Along with the increase of incidence, the position of separation is ahead displaced and nearer to the leading edge. It means that the followed turbulent region is bigger. Losses due to separation depend not also on its position but its expansion and the form of followed turbulent region. Once the separation occurs, the lift coefficient decreases.

- Beside boundary layers totally being laminar, in any case, there is always a laminar flow part at the beginning before transferring to turbulent flow part. Therefore, it is necessary to take suitable conditions of geometry, incidence, and Reynolds number in order to avoid a laminar separation.

- For incompressible flows, beside the external velocity gradient depending on the geometry and the incidence, the higher turbulent ratio is, the lower separation risk is. However, this is not verified for compressible flows becoming to transonic regime with the interaction between boundary layer and shock wave. In next works, we will present results of studies on separation phenomenon in transonic flows under the influence of Mach number, also studies on aerodynamics of wind turbines.

\section{REFERENCES}

[1] Comolet R. et Bonnin J., Mécanique expérimentale des fluides, tome 3, Editeur Dunod, Paris, 2003.

[2] Hoang Thi Bich Ngoc, Vu Manh Cuong, Nguyen Manh Hung, Calculating aerodynamic forces on wing system of subsonic airplanes, Journal of Science 83 Technology - ISSN 0868-3980, $N^{\circ}$ 48-49, (2004) $119-123$.

[3] Hoang Thi Bich Ngoc, Le Hong Chuong, Numerical calculations by solving full potential equations, Proceedings of National Conference on Engineering Mechanics and Automation, Hanoi, (2006) 171 - 180.

[4] Hoang Thi Bich Ngoc, Bui Tran Trung, Simulation of transonic flows around profiles under invicid and viscous flow theories, Proceedings of $8^{\text {th }}$ National Conference on Mechanics, Hanoi, (2007) 379 - 389.

[5] Hoang Thi Bich Ngoc, Nguyen Manh Hung, Velocity measurements on profile by means of Laser measurer, Proceedings of national Conference on Metrology, Hanoi, (2010) 438 - 448.

[6] Riegels F. W., Aerofoil sections, Butterworths Pub., London, (1961). 